\section{First diagonal artery arising from the left main coronary artery: A rare anomaly}

A 50-year-old man was admitted with complaints of Canadian Cardiovascular Society class III angina precipitated by exertion. He had inferior wall myocardial infarction (IWMI) a month back and received intravenous thrombolysis with alteplase. His electrocardiogram showed an incomplete right bundle branch block with 0 waves in II, III, and aVF leads (Fig. 1). Transthoracic echocardiogram showed mild left ventricular dysfunction with an ejection fraction of $45 \%$ and hypokinesia in inferior and lateral walls.

Subsequently, he was taken for a coronary angiogram, which revealed a significant stenosis in the left circumflex artery (LCX) and an anomalous diagonal artery originating from the distal left main coronary artery (LMCA) (Fig. 2). This anomalous diagonal artery was diseased, with a significant obstruction, and resulted in a lateral wall injury. Other vessels, including the left

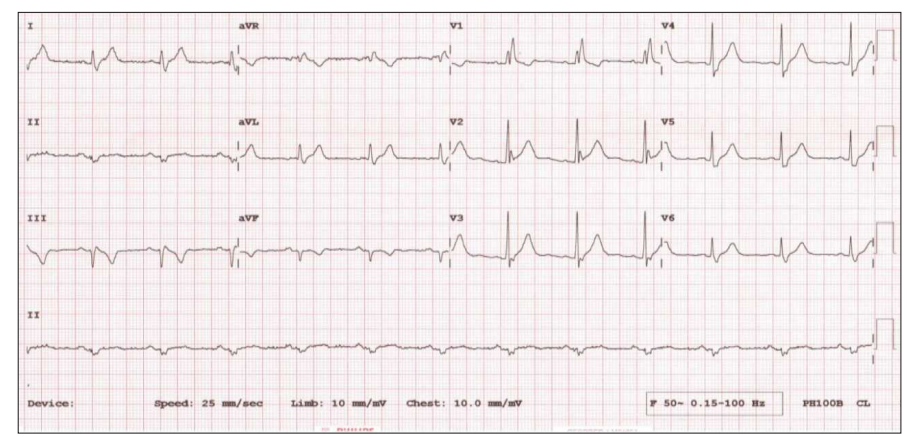

Figure 1. Baseline electrocardiogram (ECG) showing normal sinus rhythm, incomplete right bundle branch block (RBBB) with 0 waves in leads II, III, and aVF
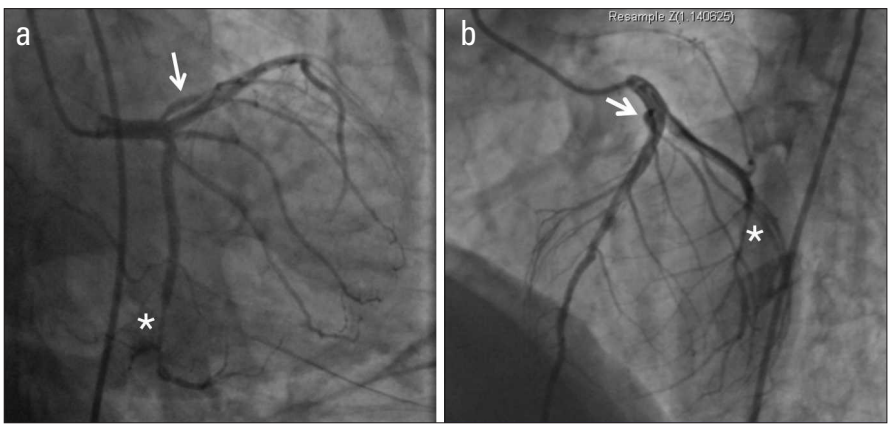

Figure 2. Invasive coronary angiography. Right anterior oblique-caudal view (a) and left anterior oblique-cranial view (b) showing diseased distal left circumflex coronary artery $\left(^{*}\right)$ and anomalous diagonal artery (arrow) arising from the distal left main artery just before the left anterior descending artery origin. There is a stenosis at the bifurcation point of this anomalous diagonal vessel
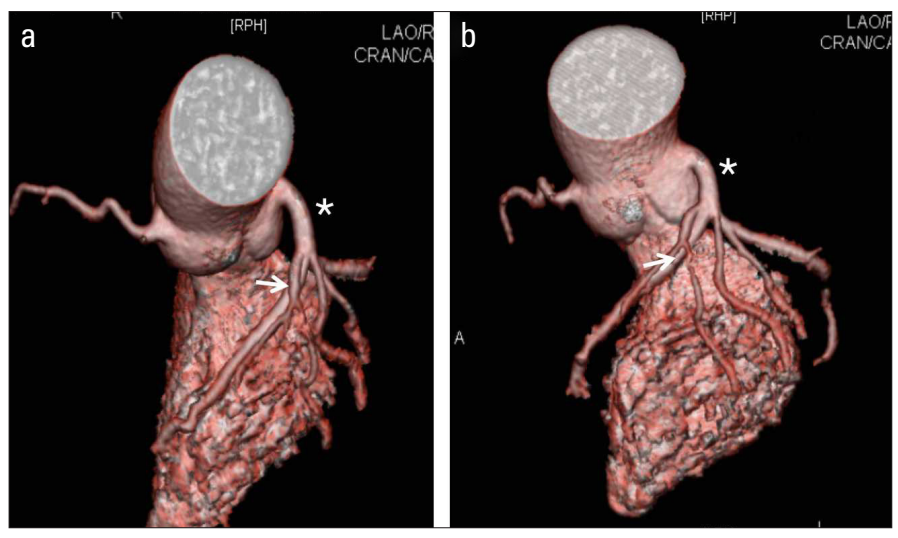

Figure 3. Cardiac computed tomographic angiogram confirms the anomalous vessel to be a diagonal artery (arrow) arising from the distal left main coronary artery (asterisk) with a stenosis at its bifurcation in modified left anterior oblique (LAO) cranial views

anterior descending and right coronary arteries, were normal. The patient was then analyzed by coronary computed tomography angiogram to delineate the anatomy better, which confirmed the anomalous vessel as the first diagonal branch, supplying the lateral wall of the left ventricle (Fig. 3).

Our index case had an exceptionally large anomalous diagonal artery with a huge area of myocardium being supplied by this vessel. IWMI was caused by the obstruction of LCx artery and lateral wall injury was the result of a significant stenosis in the large anomalous diagonal artery originating from LMCA. This anomaly of diagonal artery origin is very rare and can pose a significant challenge in coronary interventions. The patient underwent a successful LCx angioplasty with a drug eluting stent; however, the coronary guidewire could not be negotiated through the anomalous diagonal artery. Hence, he was managed conservatively for diagonal artery stenosis and remained stable at 1-year follow up.

Informed consent: Informed consent was obtained from the patient.

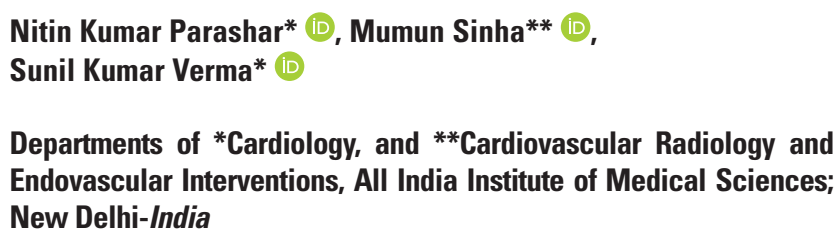

Departments of *Cardiology, and ${ }^{* *}$ Cardiovascular Radiology and Endovascular Interventions, All India Institute of Medical Sciences; New Delhi-India

Address for Correspondence: Sunil Kumar Verma, MD,

Department of Cardiology, All India Institute of Medical Sciences,

New Delhi-India

Phone: +919891039896

E-mail: ksunilverma02@gmail.com

CCopyright 2021 by Turkish Society of Cardiology Available online at

www. anatoljcardiol.com

DOI:10.5152/AnatolJCardiol.2021.539 